\title{
Combination of immune check inhibitor and immunomodulatory arabinomannan extracted from Mycobacterium tuberculosis: A case report
}

\author{
KUNIHIKO KOBAYASHI, KYOICHI KAIRA, HIDETOSHI IEMURA, \\ SHUN SHINOMIYA, KOSUKE HASHIMOTO, YU MIURA, AYAKO SHIONO, \\ FUYUMI NISHIHARA and HIROSHI KAGAMU \\ Department of Respiratory Medicine, Saitama International Medical Center, \\ Saitama Medical University, Saitama 350-1298, Japan
}

Received March 5, 2021; Accepted July 9, 2021

DOI: $10.3892 / \mathrm{mco} .2021 .2390$

\begin{abstract}
The present study selected two patients with lung cancer and epidermal growth factor receptor $(E G F R)$ mutations who were treated with a programmed cell death protein 1 (PD-1) antibody and an immunomodulatory arabinomannan extracted from Mycobacterium tuberculosis. In the first case, a 67-year-old female was diagnosed with lung adenocarcinoma with an EGFR mutation (exon 19 deletion) and Stage IVB disease. Initial treatment with an EGFR mutation-targeted tyrosine kinase inhibitor (TKI), erlotinib, demonstrated a partial response. After disease progression this was followed by carboplatin and pemetrexed with bevacizumab, and re-challenged by erlotinib plus bevacizumab; however, the tumor eventually progressed. Subsequently, the patient was treated with immunomodulatory arabinomannan for 3 months. Immediately after, she was treated with nivolumab and showed a partial response. In the second case, a 57-year-old male with a history of smoking was diagnosed with stage IVB pulmonary adenocarcinoma with an EGFR mutation (exon 19 deletion). He was treated with afatinib, followed by osimertinib when a $T 790 M$ mutation was identified later. After disease progressed with TKIs, cisplatin plus pemetrexed and re-challenge with erlotinib plus bevacizumab were administered subsequently. Nivolumab was administered for recurrent disease. Although he experienced tumor remission, regrowth of the tumors was observed. Under continuing nivolumab, he was treated by palliative irradiation treatments to the right pelvic bone metastasis and left adrenal metastasis
\end{abstract}

Correspondence to: $\operatorname{Dr}$ Kunihiko Kobayashi, Department of Respiratory Medicine, Saitama International Medical Center, Saitama Medical University, 1397-1 Yamane, Hidaka, Saitama 350-1298, Japan

E-mail: kobakuni@saitama-med.ac.jp

Key words: immune adjuvant, nivolumab, Mycobacterium tuberculosis, lung cancer, immune checkpoint inhibitor with immunomodulatory arabinomannan. A chest computed tomography scan showed a reduction in the sizes of the primary site and pulmonary metastases, with a decreasing trend of carcinoma embryonic antigen. Overall, these cases may indicate that the immune adjuvant actions of immunomodulatory arabinomannan extracted from Mycobacterium tuberculosis improves the effect of PD-1 antibody treatments.

\section{Introduction}

Sequencing of DNA to identify polymorphisms has catalyzed the quest for protein kinase 'driver' mutations, which contribute to the transformation of a normal cell to a proliferating cancerous cell. Approximately one-third of patients in Asian populations with non-small cell lung cancer (NSCLC) harbor epidermal growth factor receptor (EGFR) mutations. For these patients, EGFR-targeted tyrosine kinase inhibitors (TKIs) have shown improved efficacy and longer progression-free survival (PFS) than standard chemotherapies (1). Currently, TKI treatment is considered the standard of care for $E G F R$-mutated NSCLC. However, most patients eventually develop resistance with a PFS from 10 months to 18.9 months $(1,2)$.

For NSCLC, recent alternative treatments include immune checkpoint inhibitors (ICIs) such as programmed cell death 1 (PD-1) antibody (nivolumab or pembrolizumab) or a programmed cell death 1 ligand (PDL-1) antibody (atezolizumab or durvalumab). ICI monotherapy with nivolumab or pembrolizumab is efficacious for NSCLC, achieving response rates of $\sim 20 \%$, with a 5-year survival rate of $\sim 15 \%$. However, EGFR-mutated NSCLC is insensitive to ICIs $(3,4)$.

Previous researchers, Coley $\mathrm{W}$ and Maruyama C, experienced immune responses to human malignant tumors by erysipelas and tuberculosis, respectively, indicating there might be a relationship between infection and cancer immunity $(5,6)$. Previously, the effect of erysipelas was partially explained via the production of interleukin (IL)-12 and tumor necrosis factor (7). Regarding tuberculosis, Maruyama (6) developed Specific Substance Maruyama (SSM), a hot-water 
extract from human bacillus tuberculosis containing polysaccharides including arabinomannan and mannan (8). SSM is an immunomodulatory agent and its carcinostatic potential was reported in 1966 (6). Later studies indicated that SSM acts as an immune adjuvant, resulting in a change from innate immunity to adaptive immunity (9-12).

No report has described ICI and immunomodulatory arabinomannan extracted from Mycobacterium tuberculosis in humans.

\section{Material and methods}

The two cases presented below gave oral consents as for presenting this case-report, and written consents were also obtained from the families. This manuscript followed a Japanese law, Act on the Protection of Personal Information.

To produce a hot-water extract from human bacillus tuberculosis, Mycobacterium tuberculosis, Aoyama B strain, grown on the surface of Sauton's media for 5 weeks at 37 degrees Celsius is diluted in distilled water. To extract polysaccharides, it is kept at $100^{\circ} \mathrm{C}$ for $120 \mathrm{~min}$, and, after filtering it to delete Mycobacterium tuberculosis, the filtrate is adjusted at the following concentrations (8). There are three types of hot water extracts from human Mycobacterium tuberculosis strain Aoyama B: SSM A at a dose of $2 \mathrm{mg}$ as D-arabinose, SSM B at a dose of $0.2 \mathrm{mg}$ as D-arabinose, and Ancer ${ }^{\circledR}, 1 \mathrm{ml}$ ampoule containing Z-100 for subcutaneous injection, at a dose of $20 \mathrm{mg}$ as D-arabinose. Ancer ${ }^{\circledR}$ has been approved in Japan as a granulocyte-macrophage (GM)-colony stimulating factor (CSF) under radiation therapy. SSM and Ancer ${ }^{\circledR}$ are supplied by Zeria Pharmaceutical Co. Ltd.

The cases were treated in clinical practice, and computed tomography (CT), positron emission tomography (PET), magnetic resonance imaging (MRI), and measurement of carcinoembryonic antigen (CEA) by a kit of CEA-ABOTT JAPAN were performed when needed in clinical practice. CT was performed by Light Speed VCT (GE, USA), and Biograph mCT 16 (Siemens Healthcare, Erlangen, Germany) was used for PET-CT using 193.8MBq of F-18 FDG.

\section{Case reports}

Case one. In 2016, a 67-year-old woman was diagnosed as NSCLC (adenocarcinoma) harboring a sensitive EGFR mutation, exon 19 deletion of the right lower lung. TNM and staging were cT1bN3M1c (lymph nodes metastasis, bone metastases, and adrenal metastasis) and Stage IVb disease. Thus, an EGFR-TKI, erlotinib, was started in February 2016, and achieved a partial response (PR). However, her disease progressed 10 months after starting the treatment. Second line treatment with carboplatin and pemetrexed with bevacizumab was immediately started, and achieved a PR. As disease progressed again and her tumor had no T790M mutation, erlotinib plus bevacizumab were administered from July 2017, and this maintained stable disease (SD). However, in December 2017, the disease progressed to the neck lymph nodes and left axillary lymph nodes with multiple bone metastases, and bilateral adrenal metastases. At this point, the patient requested to be treated with SSM of her own free will. Between March and May 2018, SSM at doses of 0.2 and 2 mg as D-arabinose were alternatively administered every other day, but the disease progressed further and the SSM treatment stopped. Immediately after it, she was treated with nivolumab as fifth line treatment from June 2018. Nivolumab after SSM treatment achieved a marked PR, resulting in the disappearance of the neck lymph nodes and the left axillary lymph nodes, and decreased the size of the primary site and bone metastases (Fig. 1). Nivolumab treatment was continued for 10 cycles until December 2018. Then, interstitial lung disease of Grade 2 common toxicity criteria occurred, and nivolumab treatment was terminated. Its efficacy continued until February 2019, and then the disease progressed. She was treated by osimertinib due to having T790M, followed by nab-paclitaxel, and died in November 2020.

Case two. A 57-year-old male with a history of smoking was diagnosed with pulmonary adenocarcinoma harboring an EGFR mutation, exon 19 deletion, in the left upper lobe (TNM and staging: cT2aN2M1c (brain metastasis and bone metastases) and Stage IVb disease, respectively) in 2015. Afatinib was initiated in November 2015, and achieved PR. Tumor recurrence and a $T 790 M$ mutation were found in December 2016; therefore, osimertinib was administered. Six months after its initiation, progressive disease was observed. Then cisplatin plus pemetrexed was administered, and he also received erlotinib plus bevacizumab. However, apparent disease progression was observed in September 2017, and cancer histology by bronchial rebiopsy was changed from adenocarcinoma to undifferentiated carcinoma. Nivolumab was administered for the recurrent disease. Although he experienced tumor remission, regrowth of the primary tumor was observed in December 2019. Adding celecoxib (400 mg/day) due to lumbar pain produced a tentative effect and chest radiography revealed a shrinkage of the primary site (13). However, his disease progressed again in both the primary site and distant metastases in October 2020. He also felt pain and disturbance of motility in the bilateral legs, and it was later diagnosed as leptomeningeal metastases by MRI. Under continuing nivolumab, he was treated with palliative irradiation to the right pelvic bone metastasis at a total dose of $20 \mathrm{~Gy}$ in 4 fractions and left adrenal metastasis at a total dose of $30 \mathrm{~Gy}$ in 10 fractions with a subcutaneous injection of Ancer ${ }^{\circledR}$ for two months. In December 2020, a chest computed tomography scan showed a reduction in the sizes of the primary site and pulmonary metastases, which were not irradiated, with a decreasing trend in carcinoma embryonic antigen (CEA) blood levels (Fig. 2). However, active treatments stopped and he moved another hospital for palliative care because of progressing leptomeningeal metastases, which made him bed rest in January 2021 and died in late January 2021, indicating that the patient with leptomeningeal metastases could survive for 3 months.

\section{Discussion}

We experienced two lung cancer patients harboring EGFR mutations in whom the immunomodulatory arabinomannan extracted from Mycobacterium tuberculosis might act as an immune adjuvant under ICI treatment. Although these cases might be considered random or serendipitous, we think these 


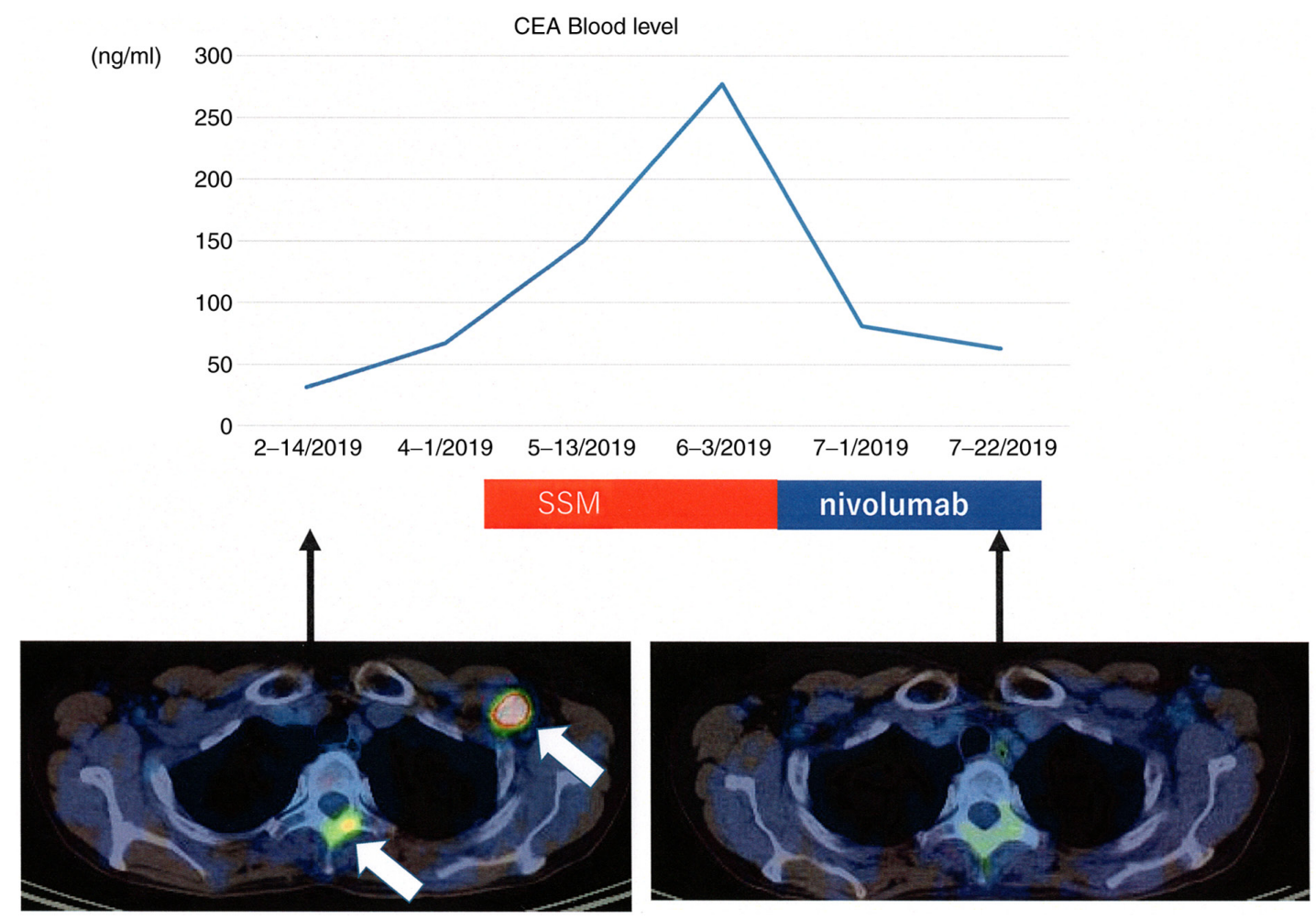

Figure 1. Case A treated with nivolumab immediately after SSM. 18F-FDG-PET before SSM treatment indicated metastases of the left axillar lymph node and vertebral bone (white arrows). After treatment with SSM followed by nivolumab, a markedly reduced uptake on FDG-PET was observed for the left axillar lymph node and vertebral bone. CEA blood levels were markedly reduced after the treatments. 18F-FDG, (18F) fluoro-2-deoxy-D-glucose; PET, positron emission tomography; SSM, Specific Substance Maruyama; CEA, carcinoembryonic antigen.
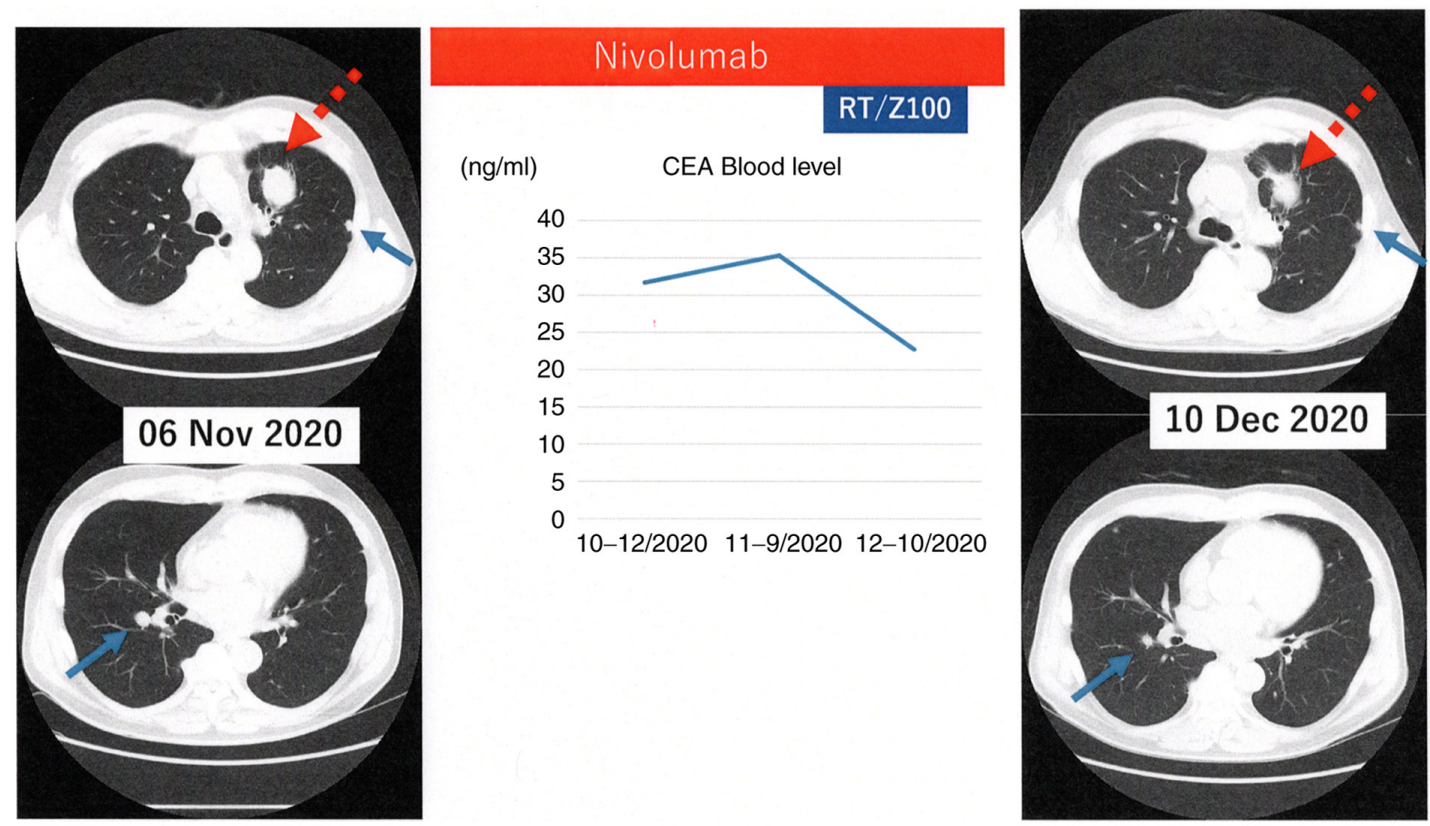

Figure 2. Case B with distant metastases irradiated with Z-100 under nivolumab treatment. Chest CT on 6 November 2020 revealed progression of the primary lung tumor (dotted arrow) and pulmonary metastases (arrows) under nivolumab treatment. After irradiation to distant metastases of the right pelvic bone metastasis and left adrenal metastasis with Z-100, the sizes of the primary lung tumor and pulmonary metastases were decreased on chest computed tomography by 10 December 2020. CEA blood level was also reduced. CEA, carcinoembryonic antigen.

cases provide interesting information. Possible mechanisms to the events described below are shown in Fig. 3.

Polymannan of tuberculosis is an immunogenic ligand for Toll-like receptors (TLRs) (14). Although signaling pathways in DCs by arabinomannan extracted from Mycobacterium tuberculosis is unknown at present, TLR ligands act as adjuvants in adaptive immune responses $(15,16)$. It was reported that SSM induced cluster of 


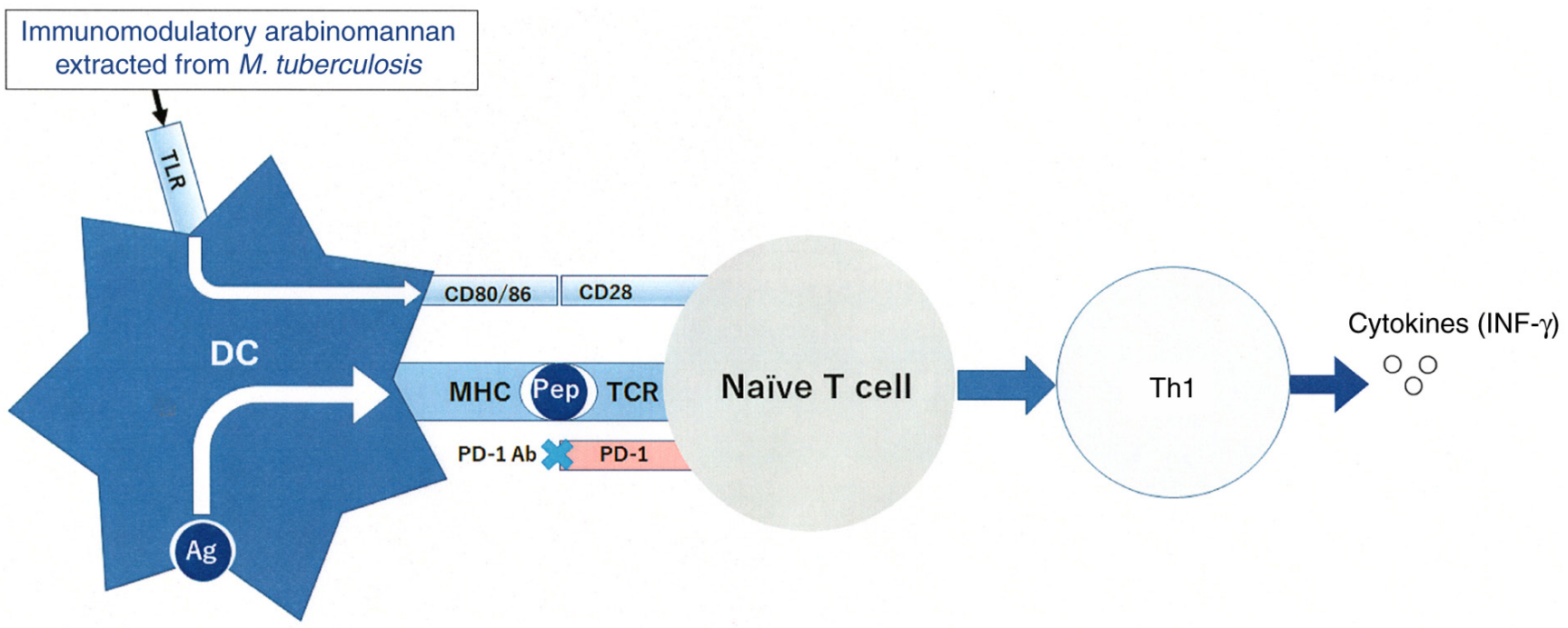

Figure 3. Enhanced immunity due to immunomodulatory arabinomannan. Immunomodulatory arabinomannan (SSM/Z-100) extracted from Mycobacterium tuberculosis induced CD80, CD86 and MHC expression on bone marrow-derived DCs. SSM/Z-100 promoted a change in helper T cell responses from a type 2 dominant state to a type 1 dominant state via the upregulation of IFN- $\gamma$ and IL-12 production. MHC, major histocompatibility complex; DC, dendritic cell; pep, peptide; PD-1, programmed cell death protein 1; Th1, T helper 1; TCR, T cell receptor; TLR, toll-like receptor; Ag, antigen.

differentiation (CD)80, CD86, and major histocompatibility complex (MHC) class II expression on bone marrow-derived dendritic cells (DCs), and that SSM treatment of mice increased the number of activated DCs in target lesions (9-12). Z-100 promoted a change in helper T-cell responses from a type 2 dominant state to a type 1 dominant state via the upregulation of interferon- $\gamma$ and IL-12 production (9-11). These reports support that arabinomannan extracted from Mycobacterium tuberculosis acts as an immune adjuvant for TLRs on DCs in cancer immunity.

Although cancer cells of EGFR-mutated NCSLC often express PDL-1 on their cell-surface, ICIs have a poor therapeutic effect $(3,4)$. This PDL-1 expression is induced directly by activated $E G F R$ signaling, not by tumor immunity (17). Therefore, PDL-1 on cancer cells harboring EGFR mutations is not exposed to any cytokines (18). Type 1 cytokine (IL-2, IFN- $\gamma$ ) production was increased in tumor-bearing mice treated with arabinomannan extracted from Mycobacterium tuberculosis $(9,10)$. In case one, in addition to SSM acting as an immunologic adjuvant, pretreatment with SSM caused tumor cells to be exposed to cytokines.

It was reported that novel proteins were generated in response to $\gamma$-irradiation, resulting in new peptides presented by MHC molecules expressed by DCs (19). Formenti et al hypothesized that 'abscopal response' might be related to the radiation-induced exposure of immunogenic mutations to the immune system (20). To produce the abscopal response, both antigen presentation by MHC and co-stimulation by an immunologic adjuvant are critical. A previous study reported the administration of Z-100 in combination with radiation showed the inhibitory action of pulmonary metastasis in tumor-bearing mice model, and prolonged survival time (11). In humans, a phase III placebo-controlled double-blind randomized trial of radiotherapy for stages IIB-IVA cervical cancer with or without Z-100 was reported a trend in the improvement of overall survival (OS) in locally advanced cervical cancer (21). The 5-year OS rate was $75.7 \%$ with Z-100 and $65.8 \%$ without $\mathrm{Z}-100$ (hazard ratio: $0.65, \mathrm{P}=0.07$ ). In our second case receiving nivolumab treatment, palliative irradiation to distant metastases was administered with Ancer ${ }^{\circledR}$, and effects on the primary site and pulmonary metastases, which were not irradiated, were observed.

In conclusion, these two cases might indicate immunomodulatory arabinomannan extracted from Mycobacterium tuberculosis has immune adjuvant effects under PD-1 antibody treatment. This treatment strategy should be validated by prospective clinical studies, and the NEJ 046A trial is currently underway.

\section{Acknowledgements}

The authors are especially grateful to Dr Hiroyuki Tajima and Dr Kenji Fukushima (Saitama Medical University, Saitama, Japan) for performing the CT/PET imaging.

\section{Funding}

No funding was received.

\section{Availability of data and materials}

The datasets used and/or analyzed during the current study are available from the corresponding author on reasonable request.

\section{Authors' contributions}

$\mathrm{KuK}$ provided the clinical data included in the text and wrote the manuscript draft. KuK, KyK, HI, SS, KH, YM, AS, FN and HK treated the two patients and interpreted the PET-CT, $\mathrm{CT}$ imaging and the laboratory test results. KyK critically revised the manuscript and modified the text. KuK and KyK confirm the authenticity of all the raw data. All authors have read and approved the final manuscript. 


\section{Ethics approval and consent to participate}

All procedures performed in the present case report were in accordance with the ethical standards of Saitama Medical University International Medical Center and with the 1964 Declaration of Helsinki and its later amendments. This manuscript followed a Japanese law, Act on the Protection of Personal Information. The two patients gave oral consent for presenting this case-report, and written consent was also obtained from the families.

\section{Patient consent for publication}

Written consent for publication was provided by the patients' families.

\section{Competing interests}

KuK received research grants from AstraZeneca Co., and Zeria Pharmaceutical Co., and received personal fees from AstraZeneca Co. KyK received personal fees from Ono Pharmaceutical Co., Boehringer Ingelheim Co., Chugai Pharmaceutical Co., Taiho Pharmaceutical Co., Eli Lilly Japan Co., Nihon Medi-Physics Co., and AstraZeneca Co. HK received research grants from AstraZeneca Co., and received personal fees from AstraZeneca Co. All other authors declare that they have no competing interests.

\section{References}

1. Maemondo M, Inoue A, Kobayashi K, Sugawara S, Oizumi S, Isobe H, Gemma A, Harada M, Yoshizawa H, Kinoshita I, et al: Gefitinib or chemotherapy for non-small-cell lung cancer with mutated EGFR. N Engl J Med 362: 2380-2388, 2010.

2. Soria JC, Ohe Y, Vansteenkiste J, Reungwetwattana T, Chewaskulyong B, Lee KH, Dechaphunkul A, Imamura F, Nogami N, Kurata $\mathrm{T}$, et al: Osimertinib in Untreated EGFR-mutated advanced non-small-cell lung cancer. N Engl J Med 378: 113-125, 2018.

3. Borghaei H, Paz-Ares L, Horn L, Spigel DR, Steins M, Ready NE, Chow LQ, Vokes EE, Felip E, Holgado E, et al: Nivolumab versus docetaxel in advanced nonsquamous non-small-cell lung cancer. N Engl J Med 373: 1627-1639, 2015.

4. Reck M, Rodríguez-Abreu D, Robinson AG, Hui R, Csőszi T, Fülöp A, Gottfried M, Peled N, Tafreshi A, Cuffe S, et al: Pembrolizumab versus chemotherapy for PD-L1-positive Non-Small-Cell lung cancer. N Engl J Med 375: 1823-1833, 2016.

5. Busch W: Einflu $\beta$ von Erysipel. Berliner Klin Wschr 3: 245-246, 1866 (In German).

6. Maruyama C: On the treatment of malignant tumor with an extract from tubercle bacilli. Jpn J Dermatol 76: 399-404, 1966 (In Japanese).

7. Tsung K and Norton JA: Lessons from Coley's Toxin. Surg Oncol 15: 25-28, 2006.
8. Kobatake H, Suekane T, Murakami Y, Niwa S, Okahira A and Kushida H: Studies on hot water extract of Mycobacterium tuberculosis. I. Structural analyses of polysaccharides (author's transl). Yakugaku Zasshi 101: 713-722, 1981 (In Japanese).

9. Oka H, Shiraishi Y, Sasaki H, Yoshinaga K, Emori Y and Takei M: Antimetastatic effect of an immunomodulatory arabinomannan extracted from Mycobacterium tuberculosis strain Aoyama B, Z-100, through the production of interleukin-12. Biol Pharm Bull 26: 1336-1341, 2003.

10. Oka H, Emori Y, Sasaki H, Shiraishi Y, Yoshinaga K and Kurimoto T: Anti-tumor mechanism of Z-100, an immunomodulatory Arabinomannan extracted from Mycobacterium tuberculosis strain Aoyama B, on pulmonary metastases of B16F10 melanoma: Restoration of helper T cell responses via suppression of glucocorticoid-genesis. Microbiol Immunol 46: 343-351, 2002.

11. Oka H, Sasaki H, Shiraishi Y, Emori Y, Yoshinaga K and Takei M: Z-100, an immunomodulatory arabinomannan extracted from Mycobacterium tuberculosis strain Aoyama B, augments anti-tumor activities of X-ray irradiation against B16 melanoma in association with the improvement of type $1 \mathrm{~T}$ cell responses. Biol Pharm Bull 27: 82-88, 2004.

12. Mitsuishi T, Kabashima K, Tanizaki H, Ohsawa I, Oda F, Yamada Y, Halifu Y, Kawana S, Kato T and Iida K: Specific substance of Maruyama (SSM) suppresses immune responses in atopic dermatitis-like skin lesions in DS-Nh mice by modulating dendritic cell functions. J Dermatol Sci 63: 184-190, 2011.

13. Kobayashi K, Kaira K and Kagamu H: Recovery of the sensitivity to Anti-PD-1 antibody by celecoxib in lung cancer. Anticancer Res 40: 5309-5311, 2020.

14. Murphy K and Weaver C: Chapters: 3-5. In: Janeway's Immunobiology. 9th edition. W.W. Norton \& Company, Inc., New York, 2017.

15. Shah RR, Hassett KJ and Brito LA: Overview of vaccine adjuvants: Introduction, history, and current status. Methods Mol Biol 1494: 1-13, 2017.

16. Reed SG, Orr MT and Fox CB: Key roles of adjuvants in modern vaccines. Nat Med 19: 1597-608, 2013.

17. Hsu PC, Jablons DM, Yang CT and You L: Epidermal Growth Factor Receptor (EGFR) Pathway, Yes-Associated Protein (YAP) and the Regulation of Programmed Death-Ligand 1 (PD-L1) in Non-Small Cell Lung Cancer (NSCLC). Int J Mol Sci 20: E3821, 2019.

18. Teng MW, Ngiow SF, Ribas A and Smyth MJ: Classifying cancers based on T-cell infiltration and PD-L1. Cancer Res 75: 2139-2145, 2015.

19. Reits EA, Hodge JW, Herberts CA, Groothuis TA, Chakraborty M, Wansley EK, Camphausen K, Luiten RM, de $\mathrm{Ru} \mathrm{AH}$, Neijssen J, et al: Radiation modulates the peptide repertoire, enhances MHC class I expression, and induces successful antitumor immunotherapy. J Exp Med 203: 1259-1271, 2006.

20. Formenti SC, Rudqvist NP, Golden E, Cooper B, Wennerberg E, Lhuillier C, Vanpouille-Box C, Friedman K, Ferrari de Andrade L, Wucherpfennig KW, et al: Radiotherapy induces responses of lung cancer to CTLA-4 blockade. Nat Med 24: 1845-1851, 2018.

21. Sugiyama T, Fujiwara K, Ohashi Y, Yokota H, Hatae M, Ohno T, Nagai Y, Mitsuhashi N, Ochiai K and Noda K: Phase III placebo-controlled double-blind randomized trial of radiotherapy for stage IIB-IVA cervical cancer with or without immunomodulator Z-100: A JGOG study. Ann Oncol 25: 1011-1017, 2014.

This work is licensed under a Creative Commons Attribution-NonCommercial-NoDerivatives 4.0 International (CC BY-NC-ND 4.0) License. 